\title{
PERCEPCIÓN ESTUDIANTIL SOBRE LA CALIDAD DE UN AMBIENTE DE APRENDIZAJE MIXTO APOYADO POR MOODLE.
}

\section{STUDENT'S PERCEPTIONABOUT QUALITY OF A BLENDED LEARNING ENVIRONMENT WITH MOODLE ASSISTANCE.}

\author{
Dr. Mario Alonso Rivero López \\ mrivero@uas.edu.mx
}

\begin{abstract}
Universidad Autónoma de Sinaloa. Facultad de Ciencias del Mar. Aula de Cómputo. Paseo Claussen s/n, Col. Pinitos, C.P. 82000. Mazatlán, Sinaloa, México
\end{abstract}

Resumen. Esta investigación se realizó con el propósito de contribuir al conocimiento acerca de la calidad del ambiente de aprendizaje ofrecido por Moodle. En la investigación participaron estudiantes del sistema semi-presencial de la licenciatura en Psicología de la Universidad Autónoma de Sinaloa, México. La encuesta COLLES (Constructivist On-Line Learning Environment Survey) (Taylor y Maor, 2000), fue utilizada para estimar diferencias en la percepción del alumnado participante acerca de la calidad ofrecida por dos cursos del sistema semi-presencial: uno ofrecido sin apoyo de Moodle y otro ofrecido con apoyo de Moodle. Los resultados revelaron que la población estudiantil prefiere realizar principalmente actividades colaborativas. Así mismo, los resultados permitieron detectar fortalezas relacionadas con la Relevancia, el Apoyo del Tutor y la Interpretación. En tanto que las áreas de oportunidad estuvieron relacionadas con el Pensamiento Reflexivo, la Interactividad y el Apoyo de los compañeros.

Palabras clave: Calidad; Aprendizaje mixto; Moodle; Percepción; COLLES.

Abstract. This research was made in order to contribute to knowledge about the quality of the learning environment offered by Moodle. In this research participated Psychology College students who were enrolled in high study level of Blended Learning system from Sinaloa University in México. The Constructivist On-Line Learning Environment Survey (COLLES) (Taylor \& Maor, 2000), was applied to estimate differences on student's perception about the quality between two educational courses of blended learning system: one offered without Moodle assistance and another one offered with Moodle assistance.The results showed that student's population preferred mainly participate in collaborative activities. Also, the results allowed detect strengths related to Relevance, Tutor Support and Interpretation. While weaknesses found were related to Reflective Thinking, Interactivity and Peer Support.

Key words: Quality; Moodle; Blended Learning; Perception; COLLES.

Píxel-Bit. Revista de Medios y Educación. No 53 Julio 2018. ISSN: 1133-8482. e-ISSN: 2171-7966. doi: http://dx.doi.org/10.12795/pixelbit.2018.i53.13 


\section{Introducción.}

Se llevó a cabo una investigación empírica con estudiantes del sistema semi-presencial de la Facultad de Psicología de la Universidad Autónoma de Sinaloa (UAS) en su zona sur, México, durante el año escolar 2016; para determinar diferencias en su percepción acerca de la calidad de dos ambientes de aprendizaje del sistema semi-presencial de educación: uno convencional y otro con apoyo de Moodle.

La encuesta COLLES (Constructivist OnLine Learning Environment Survey) (Taylor y Maor, 2000) fue el instrumento empleado para la recopilación de los datos sobre la percepción que el alumnado tuvo acerca de los ambientes de aprendizaje mencionados, con respecto a los criterios de calidad: relevancia profesional, pensamiento reflexivo, interactividad, apoyo del tutor, apoyo de compañeros y la interpretación de significados, en dos niveles de percepción: el Preferido (la expectativa) y el Real (la realidad percibida por el alumnado).

El instrumento COLLES fue diseñado para ayudar a evaluar empíricamente cuestiones clave acerca de la calidad de un ambiente de aprendizaje en línea desde la perspectiva del constructivismo social (Taylor y Maor, 2000).

Acerca del sistema semi-presencial de educación universitaria, Cladellas y Martín (2010), señalaron que se diferencia principalmente de la modalidad docente tradicional en que la mayor parte de la docencia se lleva a cabo vía on line, como por ejemplo en Moodle, Wikis o Campus virtuales, con la posibilidad de realizarse desde cualquier lugar que disponga de internet y en cualquier momento; por lo que, de acuerdo con estos autores, la modalidad semipresencial no tiene las limitaciones temporales presentes en la modalidad tradicional o presencial.

En tanto que Abasto (2009), consideró que éste sistema requiere el diseño de actividades que afiancen competencias que un docente no podría evaluar al no estar en contacto presencial semanal con el alumnado, y que quienes prefieren ésta modalidad son principalmente personas cuya ocupación laboral impediría el cursado presencial tradicional de las asignaturas.

En la Facultad objeto de la investigación, el sistema semi-presencial de educación se adoptó desde el año 2001, como una respuesta a la solicitud de un sector de la población que por cuestiones de tipo laboral no podía asistir al sistema educativo presencial; sin embargo, aspiraba a concluir estudios de nivel superior (Universidad Autónoma de Sinaloa-Facultad de Psicología, 2017).

Por otro lado, la plataforma Moodle universitaria opera desde el año 2001 dando cobertura a todas sus unidades académicas. De acuerdo con Dougiamas (2001), Moodle es una plataforma de aprendizaje que proporciona a educadores, administradores y estudiantes un sistema único, robusto y seguro para crear ambientes de aprendizaje personalizados.

Sin embargo, de acuerdo con información proporcionada por integrantes de la planta docente, la estrategia didáctica practicada regularmente en el sistema semi-presencial de la Facultad de Psicología de la UAS, comprende una reunión presencial mensual a lo largo de un semestre y no se utiliza Moodle para mediar la comunicación entre docentes y estudiantes entre una sesión presencial y la siguiente; de tal manera que al inicio de cada semestre, el/la docente organiza al alumnado en equipos de trabajo asignando a uno de sus integrantes como líder del equipo. Las funciones de cada líder de equipo consisten 
en establecer comunicación con el/la docente mediante el correo electrónico o mediante llamadas telefónicas; reportar al docente los avances y las dudas de los integrantes de equipo; y comunicar a éstos las observaciones del docente.

Por lo anterior, se planteó que la estrategia didáctica descrita representaba un problema que requería una investigación empírica, puesto que existía la posibilidad de que esa práctica dificultara la guía y el seguimiento del docente hacia la totalidad del alumnado.

Es así que, la propuesta de medidas que contribuyan a mejorar las prácticas didácticas del sistema semi-presencial de la Facultad de Psicología de la UAS como resultado de una investigación empírica, justificaron el presente trabajo.

Para ésta investigación se plantearon las siguientes hipótesis: 1) Existen diferencias en la percepción del alumnado acerca de la calidad entre dos ambientes de aprendizaje del sistema semi-presencial, uno convencional y otro mediado por Moodle. 2) La comunicación entre alumnado y docentes mediada por Moodle contribuye a mejorar la calidad de la experiencia de aprendizaje del alumnado en ese sistema. 3) Las actividades autónomas realizadas por el alumnado en Moodle fomentan la lectura, el diálogo reflexivo, la escritura y el trabajo colaborativo.

La investigación se enfocó en aquellos elementos del ambiente estudiantil que se encontraban bajo el control del investigador tales como: los contenidos, las actividades y los recursos incluidos en la plataforma Moodle; y el acceso del alumnado y docentes a la plataforma virtual de la universidad (Dougiamas y Taylor, 2002).

Otras variables del ambiente estudiantil que estuvieron fuera del control del investigador fueron la predisposición de los estudiantes (al internet, a la educación a distancia, al constructivismo, etc.); la cultura a la que pertenecen; su nivel socio-económico; el hardware y el sistema operativo que utilizaron; la calidad de su conexión a internet (disponibilidad y ancho de banda) y el número de estudiantes que optaron por matriculase en la asignatura bajo estudio (Dougiamas y Taylor, 2002).

Así mismo, la investigación estuvo delimitada por las prácticas educativas y por la interacción y el rol que asumieron docentes $\mathrm{y}$ alumnado en un sistema educativo semipresencial, donde la comunicación entre los actores estuvo mediada por Moodle.

\section{Método.}

La investigación tuvo un carácter cuantitativo (McMillan y Schumacher, 2005) porque el análisis de los datos recolectados se realizó mediante estadística descriptiva e inferencial.

Adicionalmente, con el propósito de caracterizar a la población estudiantil, se aplicó una encuesta a todo el alumnado con la que se recabo información acerca de su composición por género, su procedencia y el tiempo que cada estudiante dedica a sus actividades, entre otros aspectos.

El instrumento COLLES fue aplicado en dos momentos: primero a un grupo de 31 estudiantes pertenecientes al nivel 7 del plan de estudios vigente, quienes recibieron sus cursos de acuerdo con la práctica didáctica descrita anteriormente, a lo que se le denominó Curso Semi-presencial Convencional (CSC). En un segundo momento, el mismo instrumento fue aplicado a un grupo de 15 estudiantes pertenecientes al nivel 8 del mismo plan de estudios, quienes se matricularon de manera voluntaria en la asignatura Prácticas 
Psicológicas I, misma que se ofreció en el sistema semi-presencial con apoyo de la plataforma Moodle, designando a éste como el Curso con Apoyo de Moodle (CAM).

Para éste trabajo, se seleccionaron niveles avanzados de estudio ( 7 y 8) a los que pertenecía el alumnado que participó en la investigación. Domínguez (2011), señaló la conveniencia de este criterio de selección porque consideró que los estudiantes más adelantados en sus estudios han tenido contacto con la mayor parte del personal docente del programa educativo y están familiarizados con diferentes estilos de enseñanza. Así mismo, Conrad (2002) considera que los estudiantes más avanzados pueden ofrecer apreciaciones más objetivas sobre el desempeño de sus facilitadores.

El instrumento COLLES disponible en la versión 2.9 de Moodle, consta de 24 preguntas con 2 niveles de percepción: Preferido y Real, organizadas en las siguientes escalas o criterios de calidad:

1) Relevancia.- ¿El aprendizaje en línea es relevante para las prácticas profesionales del alumnado?

2) Reflexión.- ¿El aprendizaje en línea estimula el pensamiento crítico y reflexivo?

3) Interactividad.- ¿En qué medida el alumnado en línea se involucra en un diálogo educativo enriquecedor?

4) Apoyo del tutor.- ¿Qué tan bien los tutores facilitan la participación del alumnado en línea?

5) Apoyo de compañeros.- ¿El alumnado en línea proporciona un apoyo sensible y alentador?

6) Interpretación.- ¿Estudiantes y tutores en línea tienen una comunicación clara?

A cada participante se le solicitó calificar su grado de acuerdo en cada una de las 24 preguntas sobre una escala Likert de 5 puntos para los niveles de percepción Preferido $y$ Real: 1 = Casi nunca; 2 = Rara vez; 3 =Alguna vez; 4 = A menudo; 5 = Casi siempre. Las puntuaciones para cada uno de los 6 criterios fueron obtenidas sumando las calificaciones de las preguntas para cada nivel de acuerdo. El rango potencial de puntación para cada escala fue de 8 a 40. Una puntuación elevada indicó un alto nivel de acuerdo hacia alguno de los criterios de calidad considerados en el instrumento COLLES.

Los resultados encontrados para los criterios de calidad del COLLES en el CSC y el CAM fueron comparados estadísticamente mediante la prueba t (Daniel, 1997), para encontrar diferencias significativas en sus niveles de percepción entre ambos cursos. Cuando se encontró diferencia significativa, se determinó por diferencia la distancia en puntos porcentuales entre los niveles de percepción Preferido y Real, con lo que se obtuvo un indicador de la percepción que el alumnado tenía acerca de la calidad de esos dos ambientes de aprendizaje.

Con respecto al punto anterior, Moreno (2007) reconoció que un aspecto esencial relacionado con la evaluación de la calidad en educación es el de la medición de la distancia que existe entre la realidad y el cumplimiento de las expectativas que se tienen con respecto a las ventajas de utilizar una determinada modalidad educativa; siendo el cumplimento de tales expectativas un parámetro ineludible de calidad real.

Así mismo, Ardila (2011) interpretó a un indicador de calidad como una forma particular, normalmente numérica, en que se mide o evalúa un determinado criterio; y consideró que los indicadores de calidad, particularmente aquellos relacionados con los procesos de formación en ambientes virtuales, están constituidos por variables susceptibles 


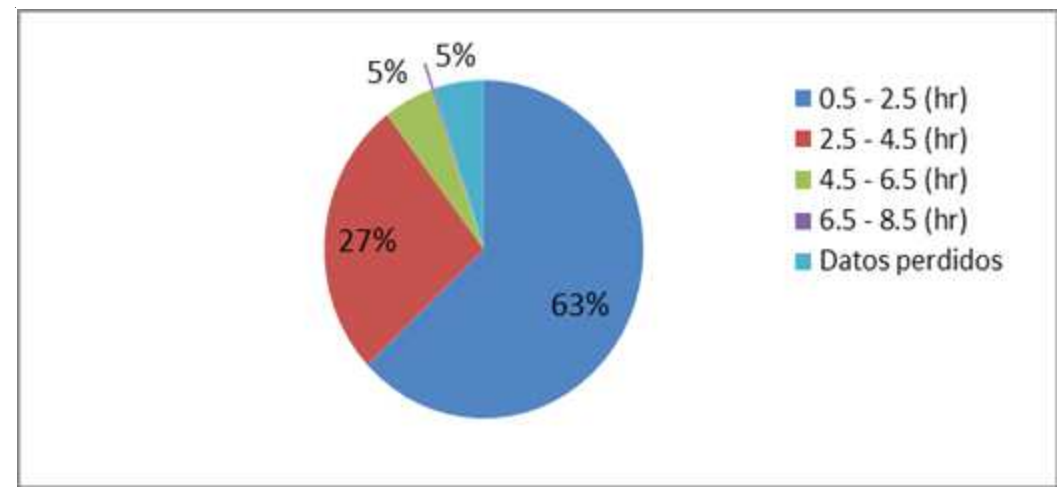

Gráfico 1. Distribución de la población estudiantil según el número de horas dedicadas a las actividades en Moodle.

de medirse y que pueden ser examinadas de manera estadística en una muestra representativa de los cursos y/o de las instituciones que ofrecen formación profesional en ambientes virtuales u on line.

La validez interna del instrumento COLLES fue determinada mediante el Coeficiente Alfa de Cronbach y el Coeficiente de Correlación de Pearson (Galotti, et.al.,1999), para cada uno de los criterios de calidad y en sus dos niveles de percepción, sin distinción de género.

Por otro lado, las sesiones presenciales realizadas los días sábado y domingo en el CAM, tuvieron el propósito de que el alumnado compartiera con el resto de la clase las experiencias de aprendizaje adquiridas durante su trabajo de campo; discutir los conceptos tomados de las lecturas y los estudios de casos disponibles en la plataforma; así como la delimitación de los avances del trabajo de campo que serían evaluados en la siguiente sesión presencial.

Al mismo tiempo, las actividades en Moodle consistieron en cuatro foros estructurados de discusión y la actividad Informe de prácticas psicológicas, realizada colaborativamente mediante la Wiki de la misma plataforma. Los recursos disponibles en Moodle estuvieron compuestos por una lectura básica, siete lecturas complementarias, diez estudios de casos, tres ligas a bases de datos arbitrados y tres tutoriales en video.

Las actividades presenciales y virtuales así como los recursos utilizados en el CAM, fueron validados por el director de la Escuela de Psicología, por el docente titular de la asignatura y por el administrador de la plataforma virtual universitaria.

\section{Resultados.}

\subsection{Caracterización de la población estudiantil.}

Al momento de realizar la investigación, la facultad objeto de estudio contaba con 357 estudiantes distribuidos en cinco niveles. Se encontró que el 63\% manifestó dedicar entre 0.5 y 2.5 horas diarias para realizar sus actividades en la plataforma (gráfico 1). Esta información se tomó en consideración cuando se diseñaron las actividades que el alumnado realizaría en la plataforma. Acorde con lo anterior, Garrison y Vaughan (2008), recomiendan no sobrecargar al estudiante con demasiadas actividades en plataforma. 


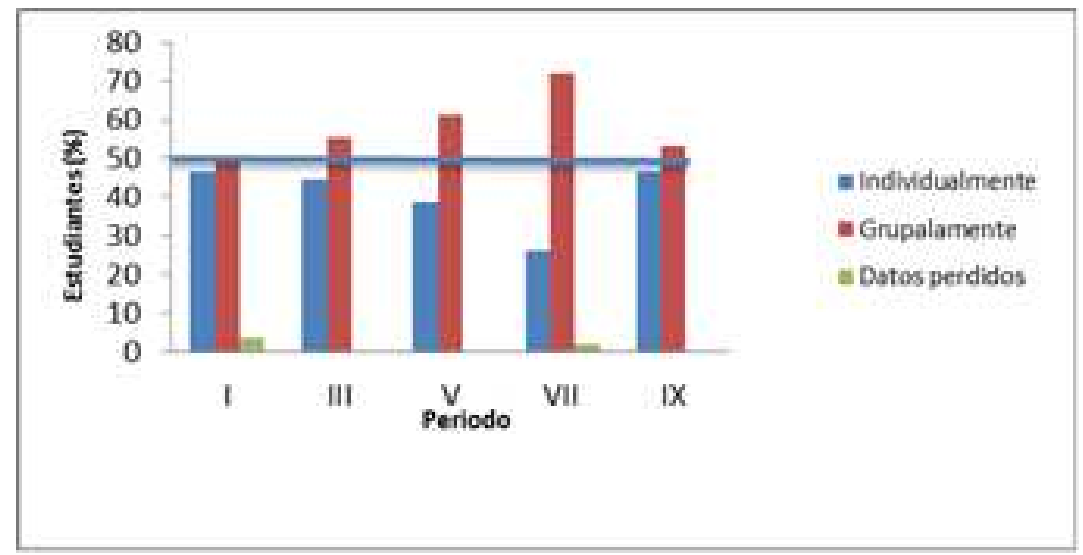

Gráfico 2. Caracterización de la población estudiantil de acuerdo con su preferencia por realizar sus actividades académicas de manera grupal o individual.

Por otra parte, al menos el 50\% del alumnado inscrito en cada período de estudio manifestó tener una preferencia por realizar sus actividades académicas de manera colaborativa (gráfico 2).

Además, el $50 \%$ de la población estudiantil declaró cubrir una jornada laboral de tiempo completo; en tanto que el $29 \%$ manifestó desempeñarse en jornadas laborales de medio tiempo. Solamente $21 \%$ de la población estudiantil declaró no trabajar al momento de contestar la encuesta.

En ese sentido, Thornton y Yoong (2011) encontraron que una de las ventajas que representa la combinación del aprendizaje en línea con las interacciones presenciales para las personas que ya se encuentran en el mundo laboral, es que una parte del tiempo que dedican al traslado físico desde su centro de trabajo al centro escolar, puede dedicarse a la realización de las actividades académicas en la plataforma.

En relación con la distribución de la población estudiantil respecto a su edad, ésta presentó un amplio rango. La edad mínima registrada fue de 19 años, en tanto que la edad máxima registrada fue de 60 años.

Por otro lado, la mayor parte de la población estudiantil $(82 \%)$ provenía de distintos municipios del estado de Sinaloa y del vecino estado de Nayarit.

Este resultado en particular se relaciona también con lo reportado por Turpo (2013), quien señaló que la convergencia de tecnología y pedagógica pueden ser una solución viable a los problemas de índole geográfico debido a la reducción de los desplazamientos físicos y el aprovechamiento del tiempo.

También se encontró que al menos $30 \%$ del alumnado inscrito en cada nivel de estudio declaró tener al menos un dependiente económico.

Por otra parte, se encontraron diversas razones que motivaron al alumnado a elegir el sistema semi-escolarizado, estando la principal razón relacionada con la posibilidad de combinar el trabajo con el estudio (gráfico 4).

De acuerdo con Turpo (2013), el modelo mixto de aprendizaje (blended learning) es elegido por las personas fundamentalmente por una pedagogía renovada, la facilidad de 


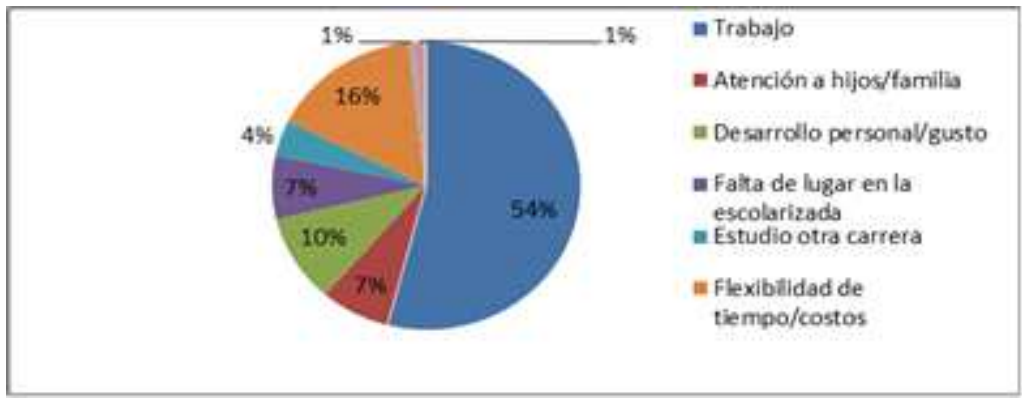

Gráfico 3. Distribución de la población estudiantil de acuerdo con el motivo de su elección por el sistema educativo semi-escolarizado.

acceso y la flexibilidad.

Sobre la disponibilidad por un dispositivo electrónico con capacidad para conectarse a internet tal como un ordenador, laptop, tablet o Smartphone; el 91\% de la población declaró contar con alguno de ellos. Esta información fue tomada en cuenta para la selección de las herramientas que el alumnado utilizaría en la plataforma Moodle y en el formato de las lecturas, con el fin de que pudieran acceder a ellos desde cualquiera de los dispositivos electrónicos con los que manifestaron contar.

En relación con el punto de acceso a internet, el $64 \%$ de la población estudiantil declaró acceder desde sus hogares, 12\% manifestó acceder desde su lugar de trabajo, otro $12 \%$ declaró acceder desde establecimientos públicos, en tanto que $8 \%$ declaró acceder desde la misma escuela.
Con base en los resultados encontrados en la caracterización de la población estudiantil, se consideró factible aplicar la metodología propuesta en la presente investigación.

\subsection{Pruebas de validez interna para el instrumento COLLES.}

Los valores del coeficiente alfa determinados para el instrumento COLLES durante la prueba de validez interna $(\mathrm{N}=31)$, proporcionaron un nivel aceptable de confiabilidad para el instrumento en sus seis criterios de calidad y en sus dos niveles de percepción, lo que le dio validez interna en su uso para la recolección de datos (Galotti, et.al., 1999), (tabla 1).

Por otro lado, no se encontró correlación significativa entre los criterios de calidad del

\begin{tabular}{|l|c|c|}
\hline \multicolumn{3}{|c|}{ Coeficiente $\boldsymbol{\alpha}$} \\
\hline & \multicolumn{2}{|c|}{ Nivel de percepción } \\
\hline \multicolumn{1}{|c|}{ Criterio de calidad } & Preferido & Real \\
\hline Relevancia & 0.8 & 0.7 \\
\hline Pensamiento reflexivo & 0.7 & 0.8 \\
\hline Interactividad & 0.6 & 0.7 \\
\hline Apoyo del tutor & 0.8 & 0.9 \\
\hline Apoyo de compañeros & 0.8 & 0.8 \\
\hline Interpretación & 0.8 & 0.8 \\
\hline
\end{tabular}

Tabla 1.- Validez interna para el instrumento COLLES con el Coeficiente Alfa. 


\begin{tabular}{|c|c|c|c|c|c|c|c|c|c|c|c|}
\hline & & \multicolumn{2}{|c|}{ Relevancia } & \multicolumn{2}{|c|}{$\begin{array}{c}\text { Pensamiento } \\
\text { Reflexivo }\end{array}$} & \multicolumn{2}{|c|}{ Interactividad } & \multicolumn{2}{|c|}{$\begin{array}{c}\text { Apoyo } \\
\text { del tutor }\end{array}$} & \multicolumn{2}{|c|}{$\begin{array}{c}\text { Apoyo de } \\
\text { compañeros }\end{array}$} \\
\hline & & $\mathrm{P}$ & $\mathrm{R}$ & $\mathrm{P}$ & $\mathrm{R}$ & $\mathrm{P}$ & $\mathrm{R}$ & $\mathrm{P}$ & $\mathrm{R}$ & $\mathrm{P}$ & $\mathrm{R}$ \\
\hline \multirow{2}{*}{ P. Reflexivo } & $\mathrm{P}$ & 0.3 & & & & & & & & & \\
\hline & $\mathrm{R}$ & & 0.2 & & & & & & & & \\
\hline \multirow{2}{*}{ Interactividad } & $\mathrm{P}$ & 0.2 & & 0.3 & & & & & & & \\
\hline & $\mathrm{R}$ & & 0.1 & & 0.3 & & & & & & \\
\hline \multirow{2}{*}{$\begin{array}{l}\text { Apoyo del } \\
\text { tutor }\end{array}$} & $\mathrm{P}$ & 0.3 & & 0.4 & & 0.4 & & & & & \\
\hline & $\mathrm{R}$ & & 0.2 & & 0.2 & & 0.2 & & & & \\
\hline \multirow{2}{*}{$\begin{array}{l}\text { Apoyo } \\
\text { compañeros }\end{array}$} & $\mathrm{P}$ & 0.2 & & 0.2 & & 0.3 & & 0.2 & & & \\
\hline & $\mathrm{R}$ & & 0.2 & & 0.2 & & 0.3 & & 0.3 & & \\
\hline \multirow{2}{*}{ Interpretación } & $\mathrm{P}$ & 0.4 & & 0.3 & & 0.2 & & 0.3 & & 0.3 & \\
\hline & $\mathrm{R}$ & & 0.2 & & 0.3 & & 0.2 & & 0.3 & & 0.4 \\
\hline
\end{tabular}

Tabla 2.- Resultados del Coeficiente de Correlación Alfa para el instrumento COLLES, entre sus escalas y para sus niveles de medición $(P=$ Preferido, $R=$ Real $)$.

\begin{tabular}{|l|c|c|c|c|c|}
\hline \multicolumn{1}{|c|}{ RELEVANCIA } & CSC & CAM & Estadisticot & Valor critico 2 colas & P(T<t) dos colas \\
\hline Mi aprendizaje se centra en asuntos que me interes & 3.74 & 4.40 & -3.2657 & 2.0555 & 0.0031 \\
\hline Lo que aprendo en importante para mi práctica profi & 4.10 & 4.60 & -2.9232 & 2.0244 & 0.0058 \\
\hline Aprendo cómo mejorar mi práctica profesional & 3.79 & 4.60 & -4.538 & 2.0195 & 0.00005 \\
\hline Lo que aprendo tiene relación con mi práctica profer & 4.00 & 4.67 & -4.3472 & 2.0301 & 0.0001 \\
\hline
\end{tabular}

Tabla 3.- Prueba t para las puntuaciones medias del criterio Relevancia entre el Curso Semi-presencial Convencional (CSC) y el Curso Semi-presencial Apoyado con Moodle (CAM).

\begin{tabular}{|l|c|c|c|c|c|}
\hline \multicolumn{1}{|c|}{ APOYO DEL TUTOR } & CSC & CAM & Estadisticot & Valor critico 2 colas & $P($ TKet) dos colas \\
\hline El tutor me estimula a reflexionar & 3.74 & 4.13 & -1.9162 & 2.0518 & 0.066 \\
\hline El tutor me anima a participar & 3.52 & 4.27 & -3.9240 & 2.0395 & 0.0005 \\
\hline El tutor ejemplifica las buenas disertaciones & 3.64 & 4.60 & -6.4474 & 2.0262 & 0.0000002 \\
\hline El tutor ejemplifica la autoreflexión crítica & 3.61 & 4.40 & -4.0255 & 2.0322 & 0.0003 \\
\hline
\end{tabular}

Tabla 4.- Prueba t para las puntuaciones medias del criterio Apoyo del tutor, entre el Curso Semi-presencial Convencional (CSC) y el Curso Semi-presencial Apoyado con Moodle (CAM).

\begin{tabular}{|l|c|c|c|c|c|}
\hline \multicolumn{1}{|c|}{ INTERPRETACION } & CSC & CAM & Estadisticot & Valor critico 2 colas & P(Tet) dos colas \\
\hline Entiendo bien los mensajes de otros estudiantes & 3.92 & 4.27 & -2.3085 & 2.0639 & 0.0299 \\
\hline Los otros estudiantes entienden bien mis mensajes & 3.57 & 4.0 & -1.3515 & 2.1009 & 0.1933 \\
\hline Entiendo bien los mensajes del tutor & 3.84 & 4.53 & -3.2285 & 2.0687 & 0.0037 \\
\hline El tutor entiende bien mis mensajes & 3.52 & 4.33 & -3.8707 & 2.0423 & 0.0005 \\
\hline
\end{tabular}

Tabla 5.- Prueba t para las puntuaciones medias del criterio Interpretación, entre el Curso Semi-presencial Convencional (CSC) y el Curso Semi-presencial Apoyado con Moodle (CAM). 


\begin{tabular}{|c|c|c|c|c|c|c|c|c|c|c|c|c|}
\hline \multicolumn{13}{|c|}{ REFPVANCA } \\
\hline \multirow[t]{3}{*}{ inem } & \multicolumn{6}{|c|}{ [1) Mi aprendizaje se ce ntraen asuintos que me interesan } & \multicolumn{6}{|c|}{ (2) lo que aprendo et importante pars mi pristica profesionsi } \\
\hline & \multicolumn{3}{|c|}{$\csc$} & \multicolumn{3}{|c|}{ CAM } & \multicolumn{3}{|c|}{$\csc$} & \multicolumn{3}{|c|}{$\cos 4$} \\
\hline & Prefetido & Res & Diferends & Preferido & Aes: & | bifferenda| & Preferido & Sed & Diferends & Preferido & Real & Diferencis \\
\hline Crei nomea & e & 0 & 0 & 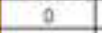 & 오 & 9 & 0 & 0 & 0 & 0 & 0 & 0 \\
\hline Raraver & 0 & 9 & 9 & 7 & 0 & 7 & 0 & 9. & 9 & 0 & 0 & 0 \\
\hline Alruns vet & 6 & 31 & 26 & 0 & 7 & 7 & is & 24 & 9 & 0 & 0 & 0 \\
\hline Amenudo & 21 & a3 & it & 49 & 47 & 7 & 12 & 33 & 20 & 27 & $\$$ & 13 \\
\hline Cos viempre & 73 & 17 & 56 & 53 & 67 & 6 & 73 & $35:$ & $3 \mathrm{~s}$ & 73 & 60 & I7 \\
\hline \multirow[t]{3}{*}{ Eem } & \multicolumn{6}{|c|}{ (3). Aprendo cono mejorze mi prictica profesionsi. } & \multicolumn{6}{|c|}{ (4) Lo Que ascendo tiene misciba con mi prictica protesional } \\
\hline & \multicolumn{3}{|c|}{$\csc$} & \multicolumn{3}{|c|}{ CAM } & \multicolumn{3}{|c|}{$\csc$} & \multicolumn{3}{|c|}{$\cos$} \\
\hline & Preferido & Real & Oterenda & Preferido & Real & Difecenda & Preterido & Real & Diferends & Peeferido & Rea! & Difeienela \\
\hline Casi wonca & 0 & 3 & 3 & 0 & 0 & 0 & 1 & 0 & 3 & 0 & 0 & 0 \\
\hline Garsvet: & 3 & 6 & 3 & 0 & 0 & 0 & 0 & 3 & 3. & 0 & 0 & 0 \\
\hline Alcunave: & II & 35 & 27 & 0 & 0 & 0 & 9 & 24 & 15 & 0 & 0 & 0 \\
\hline Amenudo & 30 & is. & $n$ & 33 & 40 & 7 & 35 & 30 & 15 & 20 & 33 & 13 \\
\hline Cas ilempre & 55 & 33 & $n$ & 67 & 60 & 7 & 53 & 24 & 29 & 80 & 67 & 17 \\
\hline
\end{tabular}

Tabla 6.- Diferencia porcentual observada en los niveles de percepción Real y Preferido entre el Curso Semi-presencial Convencional y el Curso Semi-presencial Apoyado con Moodle, para la escala Relevancia.

\begin{tabular}{|c|c|c|c|c|c|c|c|c|c|c|c|c|}
\hline \multicolumn{13}{|c|}{ AFONO DEE TuTCS } \\
\hline \multirow[t]{3}{*}{ item } & \multicolumn{6}{|c|}{ (1) B tuter me ostimela a reflexionar- } & \multicolumn{6}{|c|}{ (a) E tutor me aniin a a particlpar. } \\
\hline & \multicolumn{3}{|c|}{$\csc$} & \multicolumn{3}{|c|}{ CAM } & \multicolumn{3}{|c|}{$\csc$} & \multicolumn{3}{|c|}{ CAM } \\
\hline & Freferido & Real & Odereneia & Preferide & Ros! & Dilereneia & Preteride & Ateal & Diferencis & Preferifo & Aeal & oifereneis \\
\hline Casinunes & 0 & 0 & 3 & 2 & $\theta$ & a & 3 & o & 3 & o & o & Q \\
\hline Paraver & 7 & $t$ & 1 & 2 & $\theta$ & 0 & 0 & A & s & $\stackrel{2}{2}$ & $\theta$ & 0 \\
\hline Alguna ver $x$ & 3 & 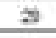 & 20 & 27 & 2t & 0 & $x$ & 4 & za & 2 & 12 & t. \\
\hline A menuso & 27 & $\Leftrightarrow$ & it & $\Leftrightarrow$ & 23 & 7 & $x$ & $\mathrm{se}$ & 4 & $\Leftrightarrow$ & 47 & e \\
\hline Canisiempre & as & 19 & 44 & 33 & 4 & 7. & 6 & 18 & 24 & \& & 49 & e. \\
\hline \multirow[t]{3}{*}{ item } & \multicolumn{6}{|c|}{ is E tutor ajemplica los buenas disertaciones. } & \multicolumn{6}{|c|}{ (4) E tuter ejemplatica la suto reflexion artica } \\
\hline & \multicolumn{3}{|c|}{$c x$} & \multicolumn{3}{|c|}{ CAM } & \multicolumn{3}{|c|}{ csc } & \multicolumn{3}{|c|}{ CAM } \\
\hline & Preteride & Real & Diformneis & Preterido & Reat & Datereneia & Preteride & Fon & Ditereneis & Preferide & Reat & Deternasia \\
\hline Casinunes & 0 & 0 & 0 & 5 & 6 & 0 & 2 & 7 & 4 & 0 & 2 & a \\
\hline Rara ien $x$ & 3 & 3 & 0 & 9 & 2 & 0 & c & 2 & 3 & ? & 0 & a \\
\hline Algona vel & 13 & $\infty$ & 27 & 0 & 7 & 7 & $v$ & 33 & 17 & $?$ & 13 & 0 \\
\hline A menusa & 43 & 4 & $a$ & 23 & 27 & e. & 2 & 45 & 17 & is & 21 & o. \\
\hline Casisiempre & 40 & 12 & 27 & 4t & 87 & 0 & $\theta$ & 17 & $x$ & $\infty$ & 63 & 7 \\
\hline
\end{tabular}

Tabla 7.- Diferencia porcentual observada en los niveles de percepción Real y Preferido entre el Curso Semi-presencial Convencional (CSC) y el Curso Semi-presencial Apoyado con Moodle, para la escala Apoyo del tutor.

Píxel-Bit. Revista de Medios y Educación. No 53 Julio 2018. ISSN: 1133-8482. e-ISSN: 2171-7966. doi: http://dx.doi.org/10.12795/pixelbit.2018.i53.13 


\begin{tabular}{|c|c|c|c|c|c|c|c|c|c|c|c|c|}
\hline \multicolumn{13}{|c|}{ NTEPREAACION } \\
\hline \multirow[t]{3}{*}{ tem } & \multicolumn{6}{|c|}{ (1) Entiendo bien los mens ajes de otros estudiantes. } & \multicolumn{6}{|c|}{ (2) Los otros estudiantes entienden bien mis mens ajes. } \\
\hline & \multicolumn{3}{|c|}{ CSC } & \multicolumn{3}{|c|}{ CAM } & \multicolumn{3}{|c|}{$\csc$} & \multicolumn{3}{|c|}{ CAM } \\
\hline & Preferido & Real & Diferencia & Preferido & Real & Diferencia & Preferido & Real & Diferencia & Prefe rido & Real & Ofierencia \\
\hline Casinunca & 0 & 0 & 0 & 0 & 0 & 0 & 0 & $\theta$ & 0 & 0 & 7 & 7 \\
\hline Rara vez & 0 & 3 & 3 & 0 & 0 & 0 & 0 & 13 & 13 & 0 & 0 & 0 \\
\hline Alguna ve $z$ & 6 & 35 & 29 & 7 & 13 & 8 & 17 & 33 & 98 & 13 & 13 & 0 \\
\hline A menudo & 52 & 45 & 7 & 47 & 47 & 0 & 45 & 42 & 5 & 33 & 47 & 14 \\
\hline Casisiempre & 42 & 18 & 28 & 47 & 42 & 7 & 38 & 13 & 25 & 52 & 33 & 20 \\
\hline \multirow[t]{3}{*}{ tem } & \multicolumn{6}{|c|}{ (3) Entiendo bien los mensajes del tutor. } & \multicolumn{6}{|c|}{ (4) $\mathrm{B}$ tutor entiende bien mis mensajes. } \\
\hline & \multicolumn{3}{|c|}{$\csc$} & \multicolumn{3}{|c|}{ CAM } & \multicolumn{3}{|c|}{$\csc$} & \multicolumn{3}{|c|}{ CAM } \\
\hline & Preferido & Real & Diferencia & Preferido & Real & Diferencia & Preferido & Real & Diferencia & Prefe rido & Real & Diferencia \\
\hline Casinunca & 0 & 0 & 0 & 0 & 0 & 0 & 0 & 3 & 3 & 0 & 0 & 0 \\
\hline Rara vez & 0 & 0 & 0 & 0 & 0 & 0 & 0 & 10 & 10 & 0 & 0 & 0 \\
\hline Alguna ve 2 & 13 & 35 & 22 & 7 & 13 & 6 & 10 & 24 & 14 & 7 & 7 & 0 \\
\hline A menudo & 38 & 42 & 4 & 20 & 20 & 0 & 38 & 88 & 3 & 33 & 53 & 20 \\
\hline Casi siempre & 50 & 23 & 27 & 73 & 87 & 6 & 32 & $?$ & 25 & (6) & 40 & 20 \\
\hline
\end{tabular}

Tabla 8. Diferencia porcentual observada en los niveles de percepción Real y Preferido entre el Curso Semi-presencial Convencional (CSC) y el Curso Semi-presencial Apoyado con Moodle, para la escala Interpretación.

instrumento COLLES (Relevancia, Pensamiento reflexivo, Interactividad, Apoyo del tutor, Apoyo de compañeros $e$ Interpretación) en sus niveles de percepción Preferido y Real, por lo que se encontró que ésos criterios de calidad en la prueba de validez fueron independientes entre sí (tabla 2).

Una vez determinada la validez interna del instrumento COLLES se procedió con la implementación del curso en modalidad semipresencial con apoyo de Moodle (CAM), de acuerdo con la metodología descrita.

\subsection{Prueba de diferencias entre el Curso} Semi-presencial Convencional (CSC) y el Curso Semi-presencial Apoyado con Moodle (CAM).

Se encontraron diferencias estadísticas significativas con el instrumento COLLES en las puntuaciones medias para los criterios de calidad Relevancia, Apoyo del Tutor e Interpretación, entre el CSC y el CAM (tablas 3,4 y 5 ).

Así mismo, las diferencias o distancias (expresadas en puntos porcentuales) observadas entre los niveles de percepción Preferido y Real para esos tres criterios de calidad, resultaron ser menores en el CAM para cada uno de los cuatro ítems comprendidos en cada criterio, lo que indicó que la percepción del alumnado acerca de la calidad del ambiente de aprendizaje semipresencial fue mayor en el CAM (tablas 6, $7 \mathrm{y}$ 8).

Los resultados encontrados por el instrumentos COLLES, constituyeron datos empíricos sobre la percepción del alumnado acerca de la calidad de dos ambientes de aprendizaje del sistema semi-presencial de la 
UAS, uno ofrecido con la práctica docente convencional y otro ofrecido con apoyo de Moodle.

\section{Discusión.}

Las pruebas de diferencias entre el CSC y el CAM, aportaron bases para aceptar las hipótesis sobre diferencias significativas en la percepción del alumnado acerca de la calidad entre ambos ambientes de aprendizaje en términos de los criterios Relevancia, Apoyo del tutor e Interpretación.

Sin embargo, los resultados encontrados para los criterios de calidad Pensamiento Reflexivo, Interactividad y Apoyo de compañeros, no presentaron diferencias significativas entre el CSC y el CAM; siendo aventurado atribuir lo anterior a algún factor en particular como la naturaleza propia del estudio, el diseño del curso, el tamaño de la muestra, la orientación que el docente responsable le dio al curso rediseñado o la situación laboral del mismo docente, entre otros posibles factores.

Por lo tanto, los resultados encontrados para los criterios Pensamiento Reflexivo, Interactividad y Apoyo de compañeros, deben ser considerados como áreas de oportunidad que requieren el desarrollo de nuevas investigaciones.

No obstante, la comunicación entre alumnado y docentes mediada por la plataforma virtual Moodle, contribuyó a mejorar la calidad de la experiencia de aprendizaje de acuerdo con la percepción del alumnado.

Es importante subrayar que las actividades autónomas tales como los foros de discusión y la Wiki para la elaboración del informe de campo, fomentaron en el alumnado la lectura, el diálogo reflexivo, la escritura y el trabajo colaborativo.

De manera similar, la guía del docente hacia el alumnado resultó beneficiada en el CAM, puesto que se facilitó la disponibilidad del material de estudio y se incrementó la comunicación del docente con el alumnado así como entre el mismo alumnado.

Es así que la problemática observada al inicio de la investigación en la Facultad objeto de estudio, puede superarse con la implementación de Moodle como un recurso mediador de la comunicación entre docentes y estudiantes.

Por otra parte, los datos recolectados con el instrumento COLLES pueden ser considerados como un referente para la realización de futuras investigaciones acerca de la interacción entre docentes y alumnado en un ambiente de aprendizaje semi-presencial mediado por una plataforma virtual.

De acuerdo con la metodología empleada, ésta podría ser replicada en otras instituciones con características similares a la Facultad objeto de la investigación, siempre que se tomen en cuenta las adecuaciones pertinentes.

En este sentido, es de particular importancia realizar una cuidadosa planeación del diseño de la instrucción que va dirigida al alumnado participante en un ambiente de aprendizaje mediado por una plataforma virtual, debido a que puede ser un elemento clave para reducir la distancia entre la expectativa y la realidad percibida por el alumnado.

Al mismo tiempo, la oferta del CAM resultó ser una experiencia de aprendizaje novedosa para el alumnado participante, quienes manifestaron haberse sentido muy satisfechos porque en este curso en particular tuvieron la oportunidad de mantenerse permanentemente en contacto con sus propios compañeros y con su profesor, situación que no se logró en elCSC. 
Finalmente, se debe mencionar el hecho de que en el CSC el tiempo disponible en las sesiones presenciales no era suficiente para que todo el alumnado presentara sus avances acerca del informe de prácticas de campo así como para que el docente aclarará todas sus dudas. Por el contrario, en el CAM el uso del recurso Wiki, ofreció al alumnado la posibilidad de elaborar de manera asincrónica y colaborativa el informe de prácticas de campo; y al mismo tiempo, el docente dispuso de un medio adecuado para ofrecer retroalimentación y seguimiento a todo el estudiantado.

\section{Fuentes de financiación.}

La investigación presentada fue financiada por la Facultad de Psicología de la Universidad Autónoma de Sinaloa en su campus ubicado en la ciudad de Mazatlán, Sinaloa, México.

\section{Referencias bibliográficas.}

Abasto, P. M. (2009). La Enseñanza de la Ecología en un nuevo Ambiente de Aprendizaje. Resultados de una Experiencia de Curso Semipresencial en la Carrera de Ingeniería Agronómica. Formación Universitaria, 2(6), 15-20. Recuperado de https://dialnet.unirioja.es/servlet/ articulo?codigo $=3122466$

Ardila, R.M. (2011). Indicadores de calidad de las plataformas educativas digitales. Educación y Educadores 14 (1), 189-206. Recuperado de http://www.redalyc.org/ articulo.oa?id=83418921011

Conrad, D. L. (2002). Engagement, Excitement, Anxiety and Fear: Learners's experiencies of starting an online course. The American Journal of Distance Education ,
16 (4), 205-226. Recuperado de http:// www.tandfonline.com/doi/pdf/10.1207/ S15389286AJDE1604_2?needAccess=true

Cladellas, P.R. y Badía, M.M. (2010). La gestión del tiempo de los profesores universitarios en función de la modalidad educativa: Sus efectos psicosociales. Revista Española de Pedagogía, 68 (246), 299. Recuperado de: https://dialnet.unirioja.es/ ejemplar/243131

Daniel, W. (1997). Bioestadística: Base para el análisis de las ciencias de la salud. México: Limusa.

Domínguez, G.N. (2011). Competencias para la Docencia en Línea. ECOESAD, México. Tésis Doctoral. Universidad da Vinci.

Dougiamas, M. (2001). Moodle: opensource software for producing internet-based courses. Recuperado de: http://moodle.com/

Dougiamas, M. \& Taylor, P. C. (2002). Interpretive analysis of an internet-based course constructed using a new courseware tool called Moodle. Proceedings of the Higher Education Research and Development Society of Australasia (HERDSA) 2002 Conference, Perth, Western Australia. Recuperado de: https:// dougiamas.com/archives/herdsa2002/

Galotti, K. M., Clinchy, B. M., Ainsworth, K., Lavin, B., \& Mansfield, A. F. (1999). ANew Way of Assessing Ways of Knowing: The Attitudes Towards Thinking and Learning Survey (ATTLS). Sex Roles, 40(9/10), 750751. Recuperado de https://link.springer.com/ article/10.1023/A:1018860702422

Garrison, D.R. \& Vaughan, N.D. (2008). Blended learning in higher education. Framework, principles and guidelines. San Francisco, CA: Jossey-Bass.

McMillan, J.H. y Schumacher S. (2005). Investigación educativa 5.a edición. Pearson Educación. Recuperado de http:// 
des.for.infd.edu.ar/sitio/upload/ McMillan_J._H._Schumacher_S._20 05._Investigacion_educativa_5_ed..pdf

Moreno, C.M. (2007). La calidad de la educación a distancia en ambientes virtuales. Apertura, 7(6), 23-24. Recuperado de http:// www.udgvirtual.udg.mx/apertura/index.php/ apertura4/article/viewFile/81/93.

Taylor, P. C. \& Maor, D. (2000). Assessing the efficacy of online teaching with the Constructivist On- Line Learning Environment Survey. In A. Herrmann and M. M. Kulski (Eds), Flexible Futures in Tertiary Teaching. Proceedings of the 9th Annual Teaching Learning Forum, 2-4 February 2000. Perth: Curtin University of Technology. Recuperado de http://clt.curtin.edu.au/events/ conferences/tlf/tlf2000/taylor.html

Thornton, K. \& Yoong P. (2011). Mixing Face-to-Face and Online Interactions in A Leadership Development Programme: A Blended Action Learning Approach. Journal of Interactive Learning Researc, 22(3), 401420. Recuperado de https:// www.learntechlib.org/p/34053

Turpo, O. (2013). Perspectiva de la convergencia pedagógica y tecnológica en la modalidad blended learning. Revista de Educación a Distancia. 39, 1-14. Recuperado de http://www.um.es/ead/red/39/

Universidad Autónoma de Sinaloa. (2017). Licenciatura en Psicología Semiescolarizada Mazatlán. Recuperado de: h t t p : / / w e b.u a s . ed u . m x / w e b / index.php? seccion $=$ oferta-educativa\&tipoc a r r e r a $=1$ i c e n c i a t u r a $\&$ a r e a $=5 \&$ op $=41 \mathrm{~d} \&$ optativas $=1 \&$ escuela $=95 \& \mathrm{c}$ arrera $=220$
Fecha de recepción: 20-07-2017

Fecha de evaluación: 08-11-2017

Fecha de aceptación: 10-01-2018 